MATEC Web of Conferences 44, 02048 (2016)

DOI: $10.1051 /$ matecconf/20164402048

(C) Owned by the authors, published by EDP Sciences, 2016

\title{
Consistently Semicontinuous Dcpo
}

\section{Di Wang, Tao Lu}

School of Mathematical Science, Huaibei Normal University, Huaibei 235000, Anhui,China

\begin{abstract}
In this paper, the concept of consistently semicontinuous depo is given,then some properties of them are discussed, especially, equivalent characterizations between consistently semicontinuous dcpo and the smallest consistently semi-prime set is given. Finally the concept of consistently semicontinuous mapping is given, and the connection between consistently dcpo and consistently semicontinuous dcpo on the base of consistently semicontinuous mapping is given.
\end{abstract}

Key words : Consistently Semi-prime set; Consistently Semicontinuous Dcpo; Consistently Semicontinuous mapping

\section{Instruction}

Because of the double background of computer science and mathematics in the continuous lattices theory [1], Since it has been widely concerned, one of the important aspect is to promote continuous lattices theory to more general order structure.In the literature [2], a new two element relation " $\Leftarrow$ " and a semi - continuous lattices are defined on a complete lattice, and some properties of continuous lattices are extended to the semi-continuous lattices.At present, there are many reports on the research of semi-continuous lattices. The related properties of semi-continuous lattices are discussed in the literature [3],[4],[5] and [6].Since the semi-continuous lattice is defined in the complete lattice, the semi- continuous study has some limitations. In order to get rid of the limitations, such as literature[7] dealing with continuous posets as the directed completion of general order structure of semi-continuous and semi-continuous lattices.in the literature [8], the lattice of semi-prime ideal is extended to partially ordered sets of semi-prime concept and in dcpo definition semi-continuity and give semi- continuous dcpo characterizations and properties, at the same time, many properties of semi-continuous lattices is extended to semi-continuous lattices. In this paper,the concept of consistently semicontinuous dcpo is given,then some properties and equivalent characterizations of them are discussed.

\section{Preparatory knowledge}

Definition $1^{[7]}$ Let $L$ be a poset, $\emptyset \neq D \subseteq L$.We say

that $D$ is a consistently directed set in a poset $L$,if $D$ is directed and for some $x \in L$ with $D \subseteq \downarrow x$.

Definition $2^{[7]}$ Let $L$ be a poset, We say that $P$ is a consistent ideal, if $P$ is a consistently directed lower set in a poset $L$.

Definition $3^{[9]}$ Let $L$ be a poset, $x \in L$, We write $X \downarrow=\{x \in P \mid x \leq y, \forall y \in S\}$, we say that is a lower bound set, $X \uparrow=\{x \in P \mid y \leq x, \forall y \in S\}$, we say that is a upper bound set.
Definition $4^{[9]}$ Let $L$ be a poset, $I$ is a ideal in a poset

$L$,we say that $I$ is a prime ideal if

$\forall x, y \in L, \exists a \in\{x, y\} \downarrow \uparrow \bigcap I$ with $x \in I$ or $y \in I$.

Definition $5^{[7]}$ Let $L$ be a poset, we say that $L$ is a consistently dcpo if every consistently directed set has the sup of in a poset $L$.

Definition $6^{[9]}$ Let $L$ be a consistently dcpo,

$I \subseteq L$ and $I$ is a consistently ideal, we say that $I$ is a

consistently prime ideal if $I$ is prime. We write it

$C R d(L)$.

\section{Consistently Semicontinuous Dcpo}

Definition 7 Let $L$ be a poset, $S \subseteq L$,we say that $S$ is a consistently semi-prime set if consistently prime ideals $\left\{P_{i}\right\}_{i \in I}$ with $S=\bigcap_{i \in I} P_{i}$.

Remark A consistently prime ideal must be a consistently semi-prime set, not the other way. It is shown in the example.

Example Let $L=\left\{x_{1}, x_{2}, x_{3} \ldots\right\} \cup\{a, b\}$, we define relationship " $\leq$ " with $\forall i \leq j, x_{i} \leq x_{j}, a, b \leq x_{i}$, $\forall i \in Z^{+}, \bigcap\left\{\downarrow x_{i}\right\}=\{a, b\}$ is a consistently semi-prime set, but $\bigcap\left\{\downarrow x_{i}\right\}=\{a, b\}$ is not directed, so is not a consistently prime ideal.

\footnotetext{
a Corresponding author: author@e-mail.org
} 
Definition 8 Let $L$ be a poset, $x, y \in L$, for every consistently semi-prime set $S$ in a poset $L$, if it has $\vee S$ and $y \leq \vee S$ with $x \in S$, we say that $x \Leftarrow_{c} y$.

We write it

$\Downarrow_{c} x=\left\{y \in L \mid y \Leftarrow_{c} x\right\}, \Uparrow_{c} x=\left\{y \in L \mid x \Leftarrow_{c} y\right\}$.

Definition 9 Let $L$ a consistently dcpo.We say that $L$ is a consistently semi-continuous depo if $\forall x \in L$, we have $x \in\left(\Downarrow_{c} x\right) \uparrow \downarrow$.

Theorem 1 Let $L$ be a consistently dcpo, then $\Downarrow_{c} x=\bigcap\{S\}=\bigcap\{P\}$, there $S$ is a consistently semiprime set always implies the existence of $\vee S$ and $x \leq \vee S, P$ is a consistently prime ideal and $x \leq \vee P$.

Proof It is immediate from the definition 7 , definition 8 and the remark.

Corollary 1 Let $L$ a consistently dcpo, $x, y \in L$ then $x \Leftarrow_{c} y$ iff for every $I \in C R d(L), y \leq \vee I$ always implies $x \in I$.

Lemma 1 Let $A, B$ be subsets of a poset $P$. If $A \subseteq B$, we have $A \uparrow \downarrow \subseteq B \uparrow \downarrow$ and $A \downarrow \uparrow \subseteq B \downarrow \uparrow$.

Proof If $A \subseteq B$, then $B \uparrow \subseteq A \uparrow, B \downarrow \subseteq A \downarrow$, therefore, $A \uparrow \downarrow \subseteq B \uparrow \downarrow$ and $A \downarrow \uparrow \subseteq B \downarrow \uparrow$.

Theorem 2 Let $L$ be a consistently dcpo, if for every $x \in L$ has consistently semi-prime set $P \subseteq \Downarrow_{c} x$ and $x \in P \uparrow \downarrow$, we have $L$ is a consistently semicontinuous depo.

Proof For every $x \in L$, it has a consistently semi-prime set $P \subseteq \Downarrow_{c} x$ by conditional knowledge and $x \in P \uparrow \downarrow$. We have $P \uparrow \downarrow \subseteq\left(\Downarrow_{c} x\right) \uparrow \downarrow$ by lemma 1, therefore we have $L$ is a consistently semi-continuous depo by definition 9.

Theorem 3 Let $L$ be a consistently dcpo. The following are equivalent:

(1) $L$ is a consistently semi-continuous dcpo;

(2) For every $x \in L, \Downarrow_{c} x$ is to
(3) For every $x \in L$, there exists the smallest consistently semi-prime set $S \subseteq \Downarrow_{c} x$ such that $x \in S \uparrow \downarrow$

Proof $(1) \Rightarrow(2)$ For every $x \in L, \Downarrow_{c} x$ is a consistently semi-prime set by theorem 1 . Since $L$ is a consistently semi-continuous dcpo, then for every $x \in L$, we have $x \in\left(\Downarrow_{c} x\right) \uparrow \downarrow$.Conversely, for every $x \in L$,when $S$ is a consistently semi-prime set and $x \in S \uparrow \downarrow$. Next, we proof that $\Downarrow_{c} x \subseteq S$.Let $y \in \Downarrow_{c} x$, since $S$ is a consistently semi-prime set, we have a family of consistently prime ideal $\left\{P_{i}\right\}_{i \in I}$ with $S=\bigcap_{i \in I} P_{i}$. For every $i \in I$, we have $x \in S \uparrow \downarrow \subseteq\left(P_{i}\right) \uparrow \downarrow=\downarrow\left(\vee P_{i}\right)$ by lemma 1. Therefore $x \leq \vee P_{i}$, and we have $y \in P_{i}$ following from $y \in \Downarrow_{c} x$. Finally we have $y \in \bigcap_{i \in I} P_{i}=S$ by any sex $i \in I$. Hence $\Downarrow_{c} x \subseteq S$.This (2) follows.

$(2) \Rightarrow(3)$ By Condition (2), we have the smallest consistently semi-prime set $S=\Downarrow_{c} x$ such that for every $x \in L$ with $x \in S \uparrow \downarrow$.

$(3) \Rightarrow(1)$ Immediate from the theorem 2 .

\section{Consistently Semicontinuous mapping}

Definition 10 Let $L, M$ be consistently depo. We we say that $f: L \rightarrow M$ is consistently prime preserving if $f$ is order preserving and every $P \in C R d(L)$ with $\downarrow f(P) \in C R d(L)$.

Definition 11 Let $L, M$ be consistently depo. We say that $f: L \rightarrow M$ is the preservation of sups about consistently ideal if $f$ is order preserving and every $P \in C R d(L)$ with $f(\vee P) \in \vee f(P)$.

Definition 12 Let $L, M$ be consistently depo. We say that $f: L \rightarrow M$ is a consistently semicontinuous mapping if for every $P \in C R d(L)$ always implies that $f(\vee P) \in \vee f(P)$ and $\downarrow f(P) \in C R d(M)$. 
Theorem 4 Let $L, M$ be consistently dcpo, a pair $(g, d)$ is a Galois connection from $L$ to $M$.If $g$ is a consistently semicontinuous mapping, then $d$ preservate relationship " $\Leftarrow_{c} "$. In other words, for every $x, y \in M, x \Leftarrow_{c} y$ implies that $d(x) \Leftarrow_{c} d(y)$ 。 Proof Let $x, y \in M, x \Leftarrow{ }_{c} y, I \in C R d(L)$ meets $d(y) \leq \vee I$, we can know that $y \leq g(\vee I)$ following from a pair $(g, d)$ is a Galois connection from $L$ to $M$. Since $g$ is a consistently semicontinuous mapping, we have $y \leq g(\vee I)=\vee g(I)=\vee \downarrow g(I)$, then $x \in \downarrow g(L)$. Therefore, there exists $z \in I$ with $x \leq g(z)$, and then $d(x) \leq d g(z) \leq z$. Finally $d(x) \in I$ and $d(x) \Leftarrow_{c} d(y)$.

Theorem 5 Let $L$ be a consistently dcpo, $M$ is a consistently semi-continuous dcpo, a pair $(g, d)$ is a Galois connection from $L$ to $M$ and for every $x \in M, \Downarrow_{c} x$ is a consistent ideal. If $g$ is a consistently semicontinuous mapping even a injection, we have that $L$ is a consistently semi-continuous dcpo. Proof Since a pair $(g, d)$ is a Galois connection from $L$ to $M, g$ is a injection, then $d$ is a surjection; we can have that for every $x \in L$, there exists $y \in M$ with $d(y)=x$. Since $M$ is a consistently semicontinuous dcpo, and for every $x \in M, \Downarrow_{c} x$ is a consistent ideal, and then $y \leq \vee \Downarrow_{c} y$. Thus $d$ is a lower adjoint and preserving of sups, we can see that $x=d(y) \leq d\left(\vee \Downarrow_{c} y\right)=\vee d\left(\Downarrow_{c} y\right)$, then we have $x \in \downarrow \vee d\left(\Downarrow_{c} y\right)$. For every $z \in M$, if $z \in \Downarrow_{c} y$ then $z \Leftarrow_{c} y$. We can know that $d(z) \Leftarrow_{c} d(y)$ by theorem 4 , that is to say $d(z) \in \Downarrow_{c} d(y)$, therefore, $d\left(\Downarrow_{c} y\right) \subseteq \Downarrow_{c} d(y)$, even more we have $x \in \downarrow \vee d\left(\Downarrow_{c} y\right) \subseteq\left(\Downarrow_{c} d(y)\right) \uparrow \downarrow=\left(\Downarrow_{c} x\right) \uparrow \downarrow$. Finally, for every $x \in L$, we have $x \in\left(\Downarrow_{c} x\right) \uparrow \downarrow$ and so $L$ is a consistently semi-continuous dcpo.

\section{References}

[1] GIERZ G, HOFMANN K H, KEIMEL K, et

al.Continuous lattices and domain [M]. Cambridge:

Cambridge University Press, 2003:1-263.

[2] ZHAO Dong-sheng Semibasis and Semibasis on Semicontinuous Lattices

[J], Algebra Univers,(37)4 (1997), 458-476.
[3] BI Han-yu, XU Xiao-quan. Semi-Scott topogy and Semi-Lawson topology on Semicontinuous Lattices [J], Fuzzy Systems and Mathematics,22(2) (2008),654-658. [4] WU Xiu-hua, LI Qing-guo. Characterizations and Function of Semicontinuous Lattices [J], JOURNAL OF MATHEMATICAL RESEARCH AND EXPOSITION, 27(3) (2007), 75-81

[5]LI Gao-lin,XU Luo-shan, CHEN Yu. Semibasis and Semibasis on Semicontinuous Lattices[J],Fuzzy Systems and Mathematics, 24(1)(2010), 51-55

[6] JIANG Guang-hao ,A Note on Semicontinuous Lattices[J],Fuzzy Systems and Mathematics, 22(1)(2008), 15-17.

[7] XU Luo-shan CONSISTENTLY CONTINUOUS POSETS AND THEIRDIRECTED COMPLETIONS [J], JOURNAL OF YANGZHOU UNIVERSITY3(1)(2000), $1-6,10$

[8]LIU Yu-lian,LI Gao-lin,XU Luo-shan Semicontinuous dcpos[J] ,Fuzzy Systems and Mathematics, 5(2) (2012), $1-5$

[9]GUO Zhi-lian,ZHAO Bin Consistently Semicontinuous Domaiins[J], Fuzzy Systems and Mathematics,2(1)(2012), 131-136 